\title{
3D visualization of convection patterns in lab-on-chip with open microfluidic outlet
}

\author{
D. Gazzola $\cdot$ E. Franchi Scarselli $\cdot$ R. Guerrieri
}

Received: 20 October 2008/Accepted: 25 February 2009/Published online: 17 March 2009

(C) The Author(s) 2009. This article is published with open access at Springerlink.com

\begin{abstract}
Open-outlet microfluidics is getting more and more attention, thanks to the generation of capillarity-driven flows which simplify the connection with the macroworld. It is known that convection flows are generated at the interface with air, i.e., the meniscus. Several works have investigated evaporation-induced convection, but its effect on particle position control in open-outlet biodevices is still not characterized. In this paper, we present the results of 3D measurement of particle traces near the meniscus in an open-outlet vertical $400 \mu \mathrm{m}$ micro-channel filled with a water-based saline solution. Using a standard optical microscope and a system of mirrors, we observe the 3D position of individual micro-beads floating in the solution, in a way akin to particle image velocimetry technique. A single vortex is generated at the meniscus and occupies the whole region under observation at a distance of $1.5-2.7 \mathrm{~mm}$ from the meniscus. The generation of the convection pattern and the vortex rotational speed are described. The convection patterns disappear when evaporation is inhibited, while both the vortex generation and the rotational speed are faster for highly saline solutions. These results are relevant to the design of biochips which require control of the particle position in a fluid since they emphasize that in open-outlet microfluidic systems not only the gravitational fall but also the convection drag must be counteracted.
\end{abstract}

Keywords Evaporation - 3D visualization . Cell position $\cdot$ Marangoni instability $\cdot$ Microfluidics

D. Gazzola $(\bowtie) \cdot$ E. Franchi Scarselli · R. Guerrieri Advanced Research Center on Electronic Systems "Ercole de Castro" (ARCES), University of Bologna, Bologna, Italy e-mail: daniele.gazzola@unibo.it

\section{Introduction}

The use of microfluidic networks with open outlets is of increasing interest in the biochip field. The main advantage is the ability to induce flows through capillarity. This gets round a complex external flow regulation system, which usually requires accurate control of the infused flow or of the inlet-outlet pressures. Furthermore, capillarity-driven microfluidics simplifies the connection to the macro-world because it eliminates external tubing, and enables monolithic devices to be designed.

Systems composed of a multitude of micro-channels with open outlets can be used for parallel assays and enable high throughput microfluidics (Juncker et al. 2002; Park et al. 2006; Zimmermann et al. 2007; Namasivayam et al. 2003). On the other hand, the use of open outlets is the cause of side effects mostly related to evaporation and contamination. In this paper, we focus on convection flows generated by evaporation; we present a measurement setup and experimental results obtained from a mixture composed of a biocompatible fluid with eukaryotic cells in it, with a view to deriving guidelines for control of cell position inside a microwell. In (Zhang et al. 2007) a thin film of an aqueous binary mixture in a large compartment was examined by the shadow-graph method to show the generation of convection patterns due to vertical temperature gradients. A vertical micro-channel is filled with water at a low temperature, incompatible with cell function, and the deflection of a cantilever is used to measure the thermocapillary flow rate dependent on the vapor-phase pressure (Ward and Duan 2004). Evaporation-induced toroidal convection flows of non-biocompatible solutions (ethanol, methanol, acetone, silicone oil, etc.) are characterized by particle image velocimetry (PIV) in vertical micro-channels (Braunsfurth and Homsy 1997; Buffone 
et al. 2004; Kamotani et al. 1994). A different flow pattern is observed in horizontal channels (Wang et al. 2008; Chamarthy et al. 2008) and the toroidal field is substituted by a single vortex in a $400 \mu \mathrm{m}$ tube because buoyancy breaks the circular symmetry of the experiment. A similar single vortex phenomenon is observed in (Buffone et al. 2005).

One big challenge in the biochips field is to control the position of cells within micro-channels. Several strategies commonly used in research have been summarized in a review by (Desai et al. 2007). They include the use of electric fields and dielectrophoresis (Abonnenc et al. 2005; Leu et al. 2005), optical trapping (Chiou et al. 2005; Nahmias et al. 2005), mechanical control through MEMSbased (Thielecke et al. 2005) or local pressure approaches (Jeong and Konishi 2009), and the use of mixed methods (Lettieri et al. 2003; Shackman et al. 2007). It is well known that all common technologies have a strong impact both on the particles and on the surrounding medium, hence limiting the applicability of these techniques. As an example, optical (Neuman et al. 1999; Leitz et al. 2002; Rasmussen et al. 2008) and electromagnetic (Seger-Sauli et al. 2005) trapping increase the local temperature through light absorption or the Joule effect, making the trapping environment uncomfortable for cells. This is particularly problematic when the biological material is required to maintain its natural function, and hence needs to be in a condition as similar as possible to its biological environment (Yang et al. 2008). Microfluidic tools used for position control are gentler because the cells are dragged by the fluid flow, which is normally induced in a region far from the cells position (Lettieri et al. 2003). We recently proposed a microfluidic approach to reduce the intensity of external forces required to maintain the position of a single cell stable in a micro-channel (Gazzola et al. 2008).

To devise guidelines for the design of biochips requiring control of particle position, in this paper we show an investigation of what actually happens in the micro-channel once an evaporation-induced flow is generated. In particular, we focus on the position of particles affected by convection patterns generated at the meniscus when the channel is filled with a typical solution for cell biology [phosphate buffer saline (PBS)]. To this end, Sect. 2 presents the measurement concept, the optical resolution, the experimental setup and the operating conditions for the observation of particle traces. In Sect. 3, we show the experimental results using eukaryotic cells in PBS. To verify that the observed flow is dependent on both evaporation and solution salinity, we show in Sect. 3.4 that inhibited evaporation prevents vortex formation, and in Sect. 3.5 that a solution with higher salinity induces vortex formation by faster dynamics. Finally, Sect. 4 presents and discusses a possible interpretation of the results based on the dependence of the surface tension on temperature and salt concentration, and gives guidelines for the control of the cell position inside open-outlet micro-wells.

\section{Materials and methods}

\subsection{Two-sided PIV}

Experiments to investigate particle position within convection flows inside a micro-channel are conducted on a custom-built device. A system of three mirrors is designed to get 3D information on the channel content, and in particular to track single particles floating in the solution. Processing the traces permits one to reconstruct particle trajectories. The idea is to observe the channel from two different directions simultaneously. The two images can be used as two projections of the channel content and can be processed to reconstruct a whole 3D image.

Particle image velocimetry (PIV) (Kamotani et al. 1994; Buffone et al. 2004) and particle tracking velocimetry (PTV) (Braunsfurth and Homsy 1997) are common techniques to image the flow field by tracking particles with laser microscopy. While PIV and PTV use microscopes with thin focal plane optics that image slices of the channel, we use a large focal plane, small magnification stereomicroscope. Herein lies the novelty of our approach: we are able to observe the whole 3D content of the channel by standard brightfield microscopy, and to obtain a complete image of single particle trajectories in time.

The three mirrors are positioned to simplify the optical access: only one microscope and one optical acquisition system are required. A first mirror (M1 in Fig. 1a) is placed at $45^{\circ}$ with the vertical axis, so that the optical path of the microscope is turned from vertical to horizontal. Two projecting mirrors (mirrors $\mathrm{M} 2$ and $\mathrm{M} 3$ ) are glued symmetrically at $67.5^{\circ}\left(90^{\circ}-22.5^{\circ}\right)$ with respect to the reflection mirror M1 as shown in Fig. 1b. The relative arrangement of mirrors and channel is designed to permit the two optical paths to reach the channel from two perpendicular directions, thus allowing for simultaneous $X-Z$ and $Y-Z$ imaging. From the two $2 \mathrm{D}$ images a $3 \mathrm{D}$ image can be reconstructed, as described in Sect. 3.1. The arrangement of the projecting mirrors with respect to the microfluidic channel is shown in Fig. 2, which also shows that the region of the micro-channel under investigation has a square section to minimize lens effects, while the rest of the channel is cylindrical.

During experiments, a movie containing the two projections is recorded. The optical data are then processed to get complete 4D information about space and time, which can be elaborated to give both the position and the velocity of the particles. 


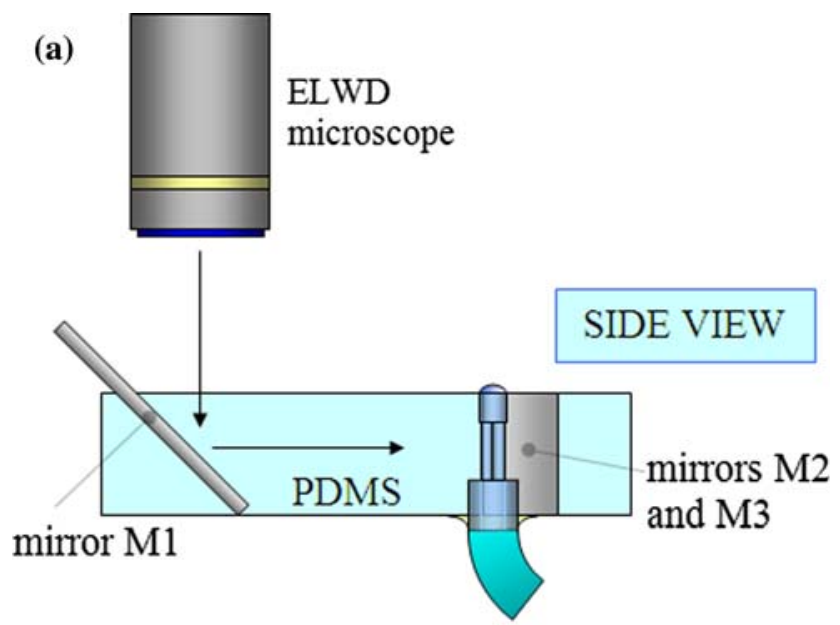

(b)

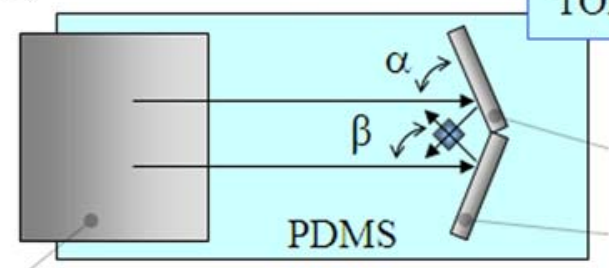

TOP VIEW

mirror M1

Fig. 1 Sketch of the micro-channel with 3D optical access: a side view, $\mathbf{b}$ top view. The values of the angles are $\alpha=67.5^{\circ}$, and $\beta=45^{\circ}$

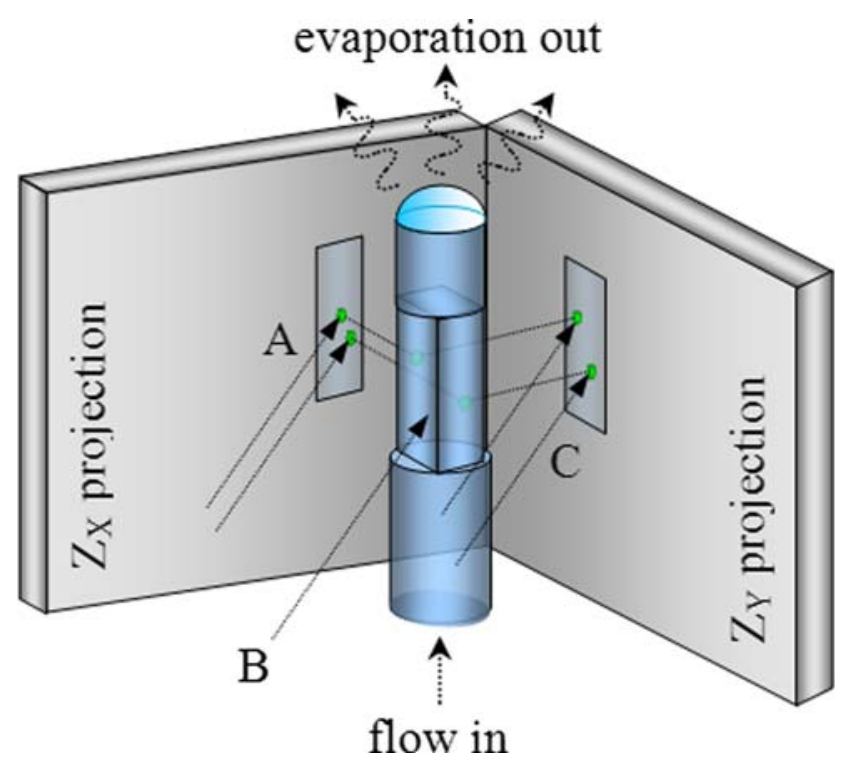

Fig. 2 The micro-channel and projecting mirrors in perspective

A typical image viewed from the microscope is shown in Fig. 3a. Recalling Figs. 2 and 3b, three views of the micro-channel are simultaneously present: at the center (zone B out of focus in Fig. 3) the channel is observed right after reflection from mirror M1; on either side it is possible to observe the $Z_{X}(\mathrm{~A})$ and $Z_{Y}(\mathrm{C})$ projections. The image shown in Fig. $3 \mathrm{a}$ is one frame of the movie processed so as to get the 3D tracks shown in Fig. 5.

\subsection{Optical resolution}

Optical investigation plays a central part in this work. The micro-channel is observed through an upright extra long working distance stereomicroscope (Zeiss Stemi DV4) coupled to a commercial camcorder (Hitachi DZ-MV270 E PAL) recording at $15 \mathrm{fps}$. Two external optical fibers illuminate the device. The overall resolution of the setup depends on the frame size regulation of the camcorder, on the resolving power of the microscope and on aberrations due to imperfections along the optical path. The theoretical resolving power of the microscope at the magnification used of $32 \times(\mathrm{NA}=0.053)$ is about $3 \mu \mathrm{m}$. However, the actual resolution of the imaging setup is made worse by the optical imperfections in the PDMS device, mostly on the mirror surfaces. The cells and beads used for the experiments are resolved when they are about $16 \mu \mathrm{m}$ apart, which should be considered the overall resolving power of the device, sufficient to observe significant movements of particles with respect to the characteristic dimensions of the observable section of the channel (Fig. 3a). The camcorder resolution $(576 \times 704$ pixel) is chosen so that each pixel corresponds to $6.36 \mu \mathrm{m}$, lower than the optical resolution. In this way there is no loss of resolution due to the camera.

The low numerical aperture of the stereomicroscope allows for a depth of field of about $200 \mu \mathrm{m}$, hence the whole channel section $(400 \mu \mathrm{m} \times 400 \mu \mathrm{m})$ is close enough to the focal plane, and all particles far from the channel walls are visible in both projections. The PDMS surface and mirror M1 are very far from the channel, so imperfections on them are not recorded. Mirrors M2 and M3 are only $1 \mathrm{~mm}$ away from the channel, and imperfections are thus visible as out of focus shadows, and do not spoil data elaboration. Obviously, a few evident defects and trapped dirt on the PDMS channel walls mask parts of the region under investigation.

\subsection{Device fabrication}

The device is composed of a micro-channel fabricated in polydimethylsyloxane (PDMS) which is contained in a glass box, and three mirrors used for optical inspection (Fig. 4). The mirrors are embedded in PDMS and are oriented at the angles described in Sect. 2.1. The fabrication steps used to build the device are described in the following list:

1. a hole is drilled on the bottom glass slide as an inlet; 
Fig. 3 a Microscope view of the micro-well. b Schematic view of the channel projections onto mirrors M2 and M3. On the left and right sides of both images, the two projections (A and B) of the channel are visible. The region of interest (ROI) corresponds to the square section of the channel and is between the two dashed lines
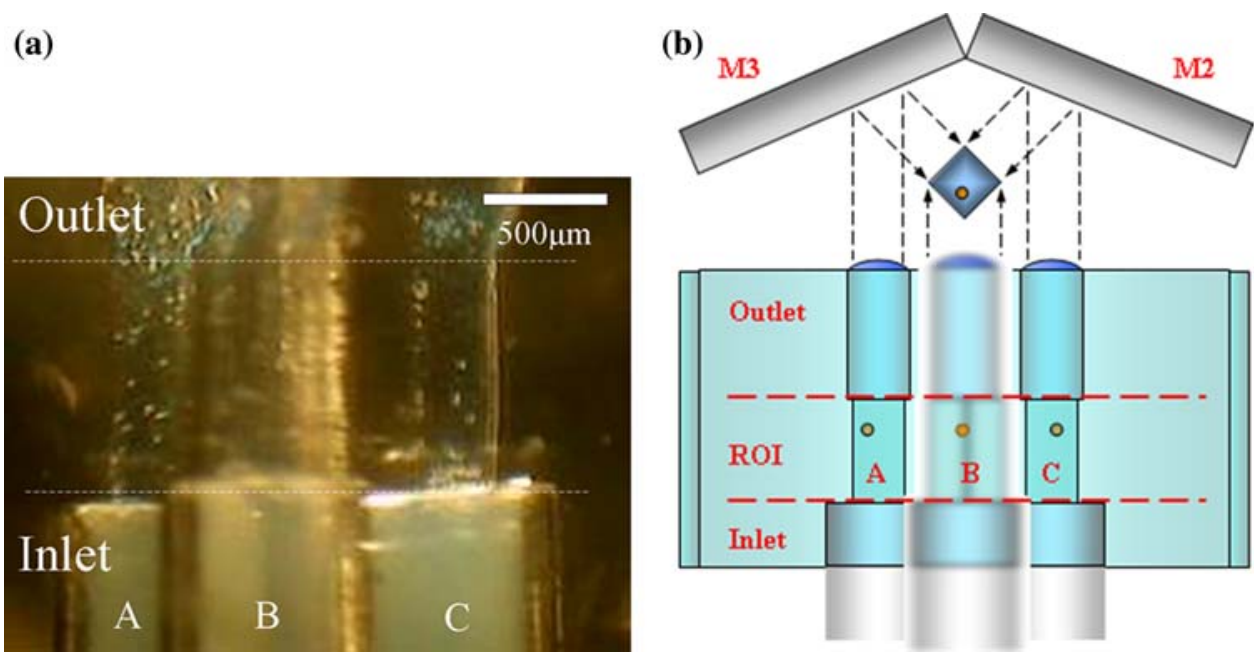

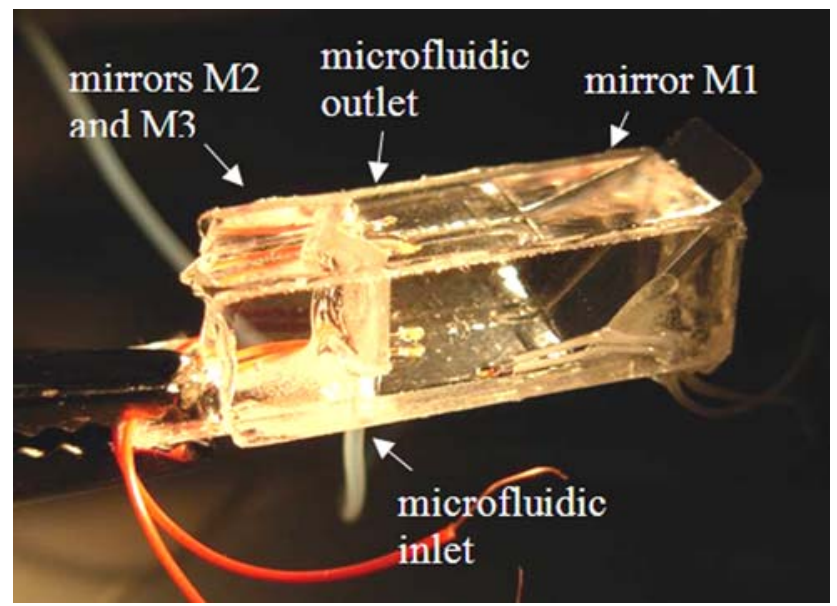

Fig. 4 Photograph of the device used for 3D optical imaging of convection flows

2. the side glass walls and mirror M1 are glued onto the bottom glass slide. Mirror M1 is glued at $45^{\circ}$ to the vertical axis, as shown in Fig. 1a;

3. the mould for the micro-channel is composed of three sections: a $400 \mu \mathrm{m}$-sided square manually polished metal pin is glued between a $500 \mu \mathrm{m}$ diameter glass capillary and a $1 \mathrm{~mm}$ external diameter PTFE tube (Borland);

4. the two mirrors M2 and M3 and the micro-channel mould are glued onto the top glass slide using a very small amount of epoxy glue, so as to be able to remove the top glass slide after polymerization. The microchannel is oriented at $45^{\circ}$ to the reflection mirror, as shown in Fig. 1b. In this way, the two optical paths reach the channel from two perpendicular directions;

5. PDMS (Sylgard 184, Dow Corning, Midland, MI) is prepared at the usual 1:10 proportions, mixed thoroughly, centrifuged at 2,000 rpm for 2 min to remove gas bubbles, poured into the mould and degassed for 30 min. Polymerization is conducted at $60^{\circ}$ overnight; 6. the glass lid and micro-channel mould are removed.

The channel mould is composed of three sections, as described above. The lower one, made from circular PTFE tubing, is designed to host the microfluidic inlet. Sealing at the inlet is assured because the mould used for this part of the channel is the same tubing as used for the inlet connection. The middle part corresponds to the region of interest (ROI), with a square section of approximately $400 \mu \mathrm{m}$. The top circular section is made of a glass capillary pulled by hand from a Pasteur pipette. Its function is to end the channel with a circular opening, minimizing non-symmetric evaporative effects. The dimensions of the three parts of the micro-channel are chosen in order to keep the section area as constant as possible: $500 \mu \mathrm{m}$ inner diameter of the inlet connection tubing, $400 \mu \mathrm{m}$ for the sides of the square section, and $500 \mu \mathrm{m}$ again for the outlet section. A sketch of the micro-channel regions is shown in Fig. 2.

The fabrication steps could be adapted for tube diameters ranging from $100 \mu \mathrm{m}$ to a few millimeters, and designed to observe either an inner part of the microchannel or a region near the outlet.

\subsection{Meniscus height control}

The solution is fed via the principle of communicating vessels: a $50 \mathrm{~cm}$ long silicone tube with a $500 \mu \mathrm{m}$ inner diameter receives solution from a $2 \mathrm{ml}$ Eppendorf tube hanging from a vertical column so that the user can adjust its height. Particular attention must be paid to avoiding air bubbles while filling the tube. Once the desired height of the meniscus is reached, the reservoir is held stable to ensure constant inlet pressure. In all the experiments 
Fig. 5 a, b The traces of two micro-beads are superposed on the actual $X-Z_{x}$ and $Y-Z_{y}$ images of the channel, where the dashed lines represent the channel walls; $\mathbf{c}$ the two traces are combined to form a reconstruction in time and space of the trajectory of the micro-beads
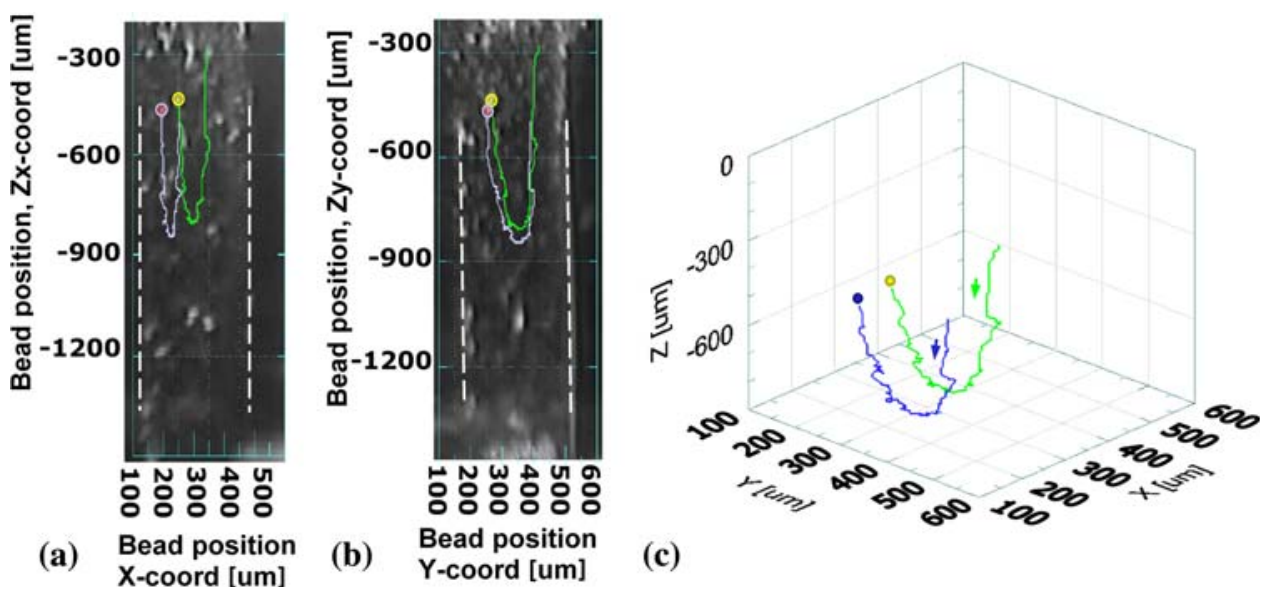

(c)

Various experiments were performed at room temperature and humidity: one set uses a PBS solution with eukaryotic cell line K562 $\left(r_{\text {part }} \sim 5 \mu \mathrm{m}, \rho_{\text {part }} \sim 1,060 \mathrm{~kg} /\right.$ $\left.\mathrm{m}^{3}\right)$. The PBS solution is an aqueous solution of $137 \mathrm{mM}$ $\mathrm{NaCl}, 10 \mathrm{mM}$ Phosphate, $2.7 \mathrm{mM} \mathrm{KCl}$, at $\mathrm{pH}$ 7.4. The second set uses a solution with an increased salt concentration $\mathrm{KCl} 0.8 \mathrm{M}\left(\rho_{\mathrm{KCl}}=1,047 \mathrm{~kg} / \mathrm{m}^{3}\right)$ with polystyrenecolored micro-beads $\left(r_{\text {bead }} \sim 5 \mu \mathrm{m}, \rho_{\text {bead }}=1,050 \mathrm{~kg} / \mathrm{m}^{3}\right)$. The measured gravitational fall is $v_{\text {grav }} \sim 1.8 \mu \mathrm{m} / \mathrm{s}$ for cells in PBS (Sect. 3.4), and $v_{\text {grav }} \sim 1 \mu \mathrm{m} / \mathrm{s}$ for micro-beads in $\mathrm{KCl} 0.8 \mathrm{M}$ solution (data not shown), and needs to be taken into consideration during data interpretation.

\section{Experiments}

\subsection{Three-dimensional particle tracking}

A custom application in LabView analyzes the movies recorded during experiments. The horizontal and vertical coordinates are called $X$ and $Z_{x}$ for the left side and $Y$ and $Z_{y}$ for the right side. The $X-Z_{x}$ and $Y-Z_{y}$ tracks are recorded for different particles of interest. In Fig. 5a, b, we show the tracks of two $10 \mu \mathrm{m}$ diameter micro-beads in $\mathrm{KCl} 0.8 \mathrm{M}$ solution. For greater clarity the tracks are superimposed on a corresponding channel image taken from the movie.

Normally more than one particle is present in the region under observation at the same time. To reconstruct the 3D trajectory of a single particle, we need to associate each $X-Z_{x}$ track with the corresponding $Y-Z_{y}$ track. When plotting the $Z_{x}$ versus the $Z_{y}$ data, the right combination is the one that shows a linear plot with an angular coefficient equal to 1 . Each point of a track is then defined by the space-time coordinates $\left(X, Y, 1 / 2\left(Z_{x}+Z_{y}\right), t\right)$, where $t$ is the time of the movie. Figure $5 \mathrm{c}$ shows a perspective of the 3D reconstruction of the tracks of Fig. 5a, b. 


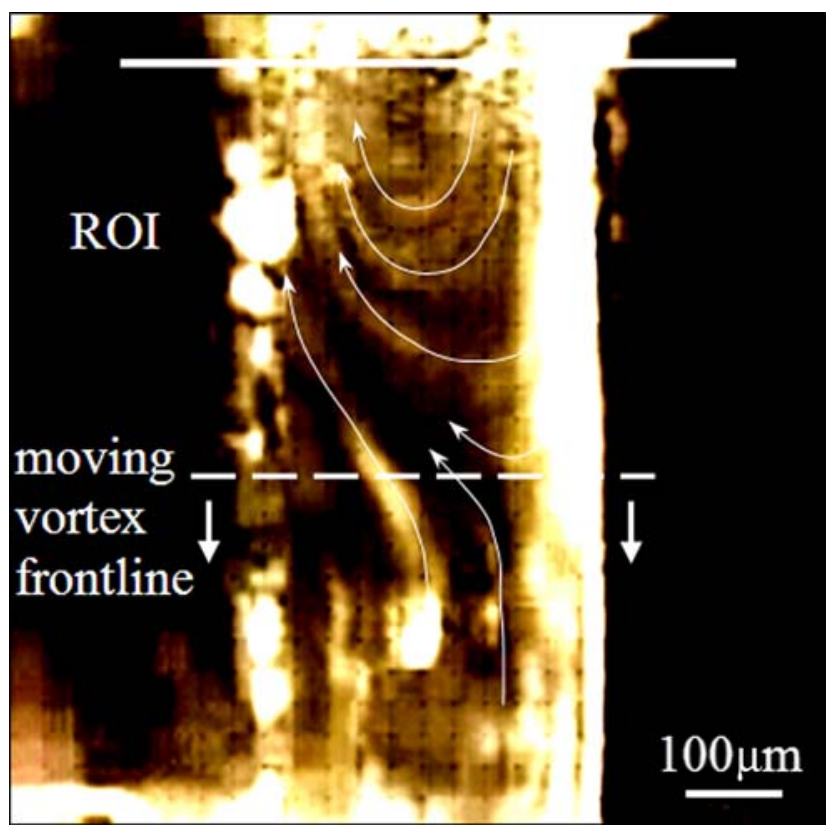

Fig. 6 Traces of the vortex flow field (arrows). The recorded images have been elaborated with a blur filter to help the reader understand the concept of vortex frontline. The frontline (dashed line) is defined as the lowest position of the traces that close in on themselves in a circular manner. The frontline moves down with time. The continuous line denotes the top of the region under observation (about $1,500 \mu \mathrm{m}$ from the outlet). The whole ROI is about $1,200 \mu \mathrm{m}$ long

\subsection{Dynamics of the vortex frontline}

Visualization of three-dimensional particle tracks is used to identify the effects of convection patterns induced by evaporation from the open outlet on particle position.

Recording is started when the channel is filled with a solution. In all measurements, a single vortex is observed, which generates from the meniscus (outside the region under observation) and extends to the inner part of the channel until it occupies the whole observed region. While the vortex formation is reproducible, its orientation varies between different experiments, but stays constant during the course of a single observation. The vortex frontline is measured directly on the row movies without any image processing by tracking single beads. When each bead reaches its minimum vertical position, the $z$-coordinate and its corresponding timestamp are recorded. Figure 6 is added for clarity's sake, so as to graphically visualize the vortex frontline as the lowest vertical position of particle traces closing in on themselves in a circular manner (the tracks above the dashed line). Figure 6 is an elaboration of a set of images from one of the two projections, applying a motion blur filter (18 frames distant 32 frames from each other) after a lever filter to increase contrast, so that the particle traces can be observed in a single picture.

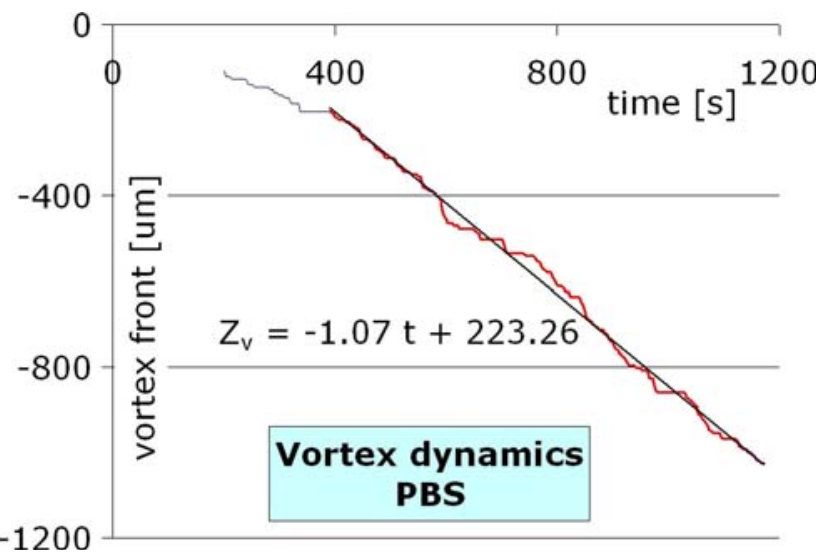

Fig. 7 Dynamics of vortex generation in PBS. The vertical position of the vortex frontline (dashed line in Fig. 6) with respect to the top of the region under observation (continuous line in Fig. 6) is plotted versus time

The graph in Fig. 7 shows typical vortex frontline dynamics in PBS, traced through eukaryotic cells.

The whole ROI is about $1,200 \mu \mathrm{m}$ long. Unexpectedly, the frontline descends at an approximately constant speed. The lack of asymptotic behavior suggests that the vortex reaches much deeper regions, probably in the order of a few centimeters.

Linear regression is performed on the linear part of the curve. The formula of the linear fit is shown on the graph in Fig. 7. The parameter of interest is the angular coefficient, as this characterizes the speed of vortex generation in the channel. The velocity of vortex generation $\left(v_{\mathrm{g}}\right)$ is hence:

$v_{\mathrm{gPBS}} \sim 1.07 \mu \mathrm{m} / \mathrm{s}$

\subsection{Characteristic vortex velocities}

As observed above, after a micro-channel fills up with solution, a single vortex spreads from the meniscus region into the channel. After several minutes, the vortex occupies the whole region under observation (i.e., $2.7 \mathrm{~mm}$ from the meniscus), so that the flow in one part of the channel is ascending, while in the other one it is descending. In laminar conditions, the profile of both the ascending and descending flows is parabolic. The velocity is maximum at the center of each flow and is almost zero at the channel walls and at the interface between the ascending and descending flows. Each particle is dragged by the local flow, so its velocity depends on its position in the channel. To compare the ascending and descending vortex velocities in different conditions, we track only the particles in the central portion of each flow region. This is the region of major interest for the purpose of this paper because we aim to calculate the additional force such as dielectrophoresis required to control the position of cells where the convective drag is maximum. 
Particle speed is measured by processing the recorded movie with the custom-designed software described in Sect. 3.1. Measurement of the velocities was performed for more than $30 \mathrm{~min}$ during which time the whole ROI was completely occupied by the vortex. During observation of a single particle the velocity value showed no significant drift. A superposition of the histograms for $v_{\text {up }}$ and $v_{\text {down }}$ in PBS, the particle velocities measured in the central portion of the ascending and descending flow regions, respectively, is shown in Fig. 8.

The mean value of the measured single particle velocities, which can be considered the characteristic upward stream velocity $\left(v_{\text {up }}\right)$, is $9.8 \mu \mathrm{m} / \mathrm{s}$, similar to the downward one $\left(v_{\text {down }}\right)$ which is $9.0 \mu \mathrm{m} / \mathrm{s}$. As described in Eq. 3 of Sect. 2.5, these values are a combination of solution velocity and particle gravitational fall. The latter is in the order of $2 \mu \mathrm{m} / \mathrm{s}$ for cells in PBS (Sect. 3.4), lower than but comparable to the measured particle velocities. This implies that the values shown in the histogram are only to be interpreted as a qualitative description of the flow field. Nevertheless, from the experimental results an average net ascending flow velocity in the order of one micrometer per second can be estimated. This value is comparable with the average velocity of evaporative flow in a micro-channel, as measured by (Gazzola et al. 2008).

\subsection{Inhibited evaporation}

The experiments show a clear effect, but a negative experiment needs to be devised if one is to assert that the vortex is triggered by evaporation at the interface. In this section, we show how the inhibition of evaporation eliminates vortex generation. The experiment is performed in PBS, and evaporation is inhibited by positioning a cap at the exit to the micro-channel so that it creates a closed region above the meniscus. Once vapor saturation is reached in the cap, evaporation stops.

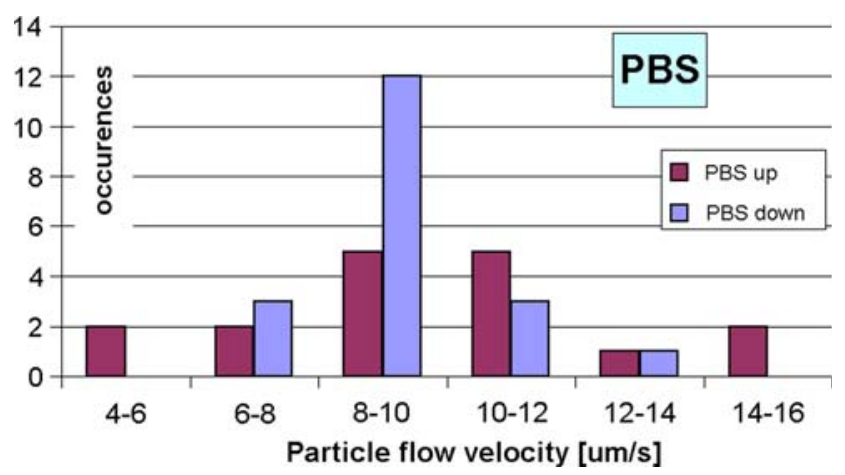

Fig. 8 Histogram of particle velocities in PBS measured in the central portion of the ascending and descending flow regions. The red tiles represent particles moving upward, and the blue ones represent particles moving downward

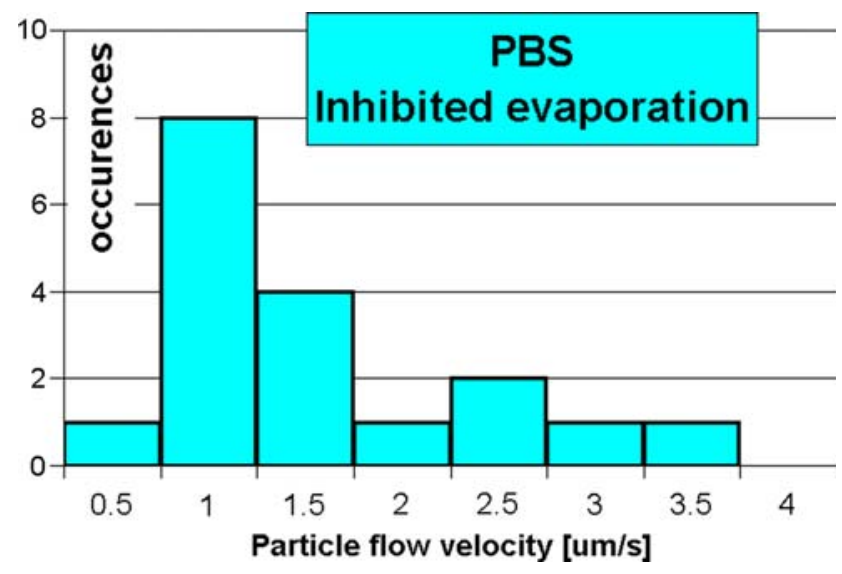

Fig. 9 Histogram of falling particle velocity in PBS once evaporation is inhibited

The cap is a circular well of $2 \mathrm{~mm}$ diameter and $1 \mathrm{~mm}$ height realized in PDMS. The small dimension of the well, chosen to minimize the volume, forces evaporation to be inhibited by vapor saturation a short time after the experiment begins $(<1 \mathrm{~min})$. In this situation, the flow is observed to be very stable for as long as $30 \mathrm{~min}$. No vortex is observed, and a homogeneous downward motion of the cells is recorded (Fig. 9).

The mean value of the measured velocities $V_{\text {grav }}$ is $1.8 \mu \mathrm{m} / \mathrm{s}$ downwards. This value is compatible with the expected gravitational fall of cells in PBS (Eq. 3).

\subsection{Increase in salt concentration}

To verify that the observed phenomena are dependent on salt concentration, the experiments were repeated with an increased salt concentration. These extreme conditions are not suitable for eukaryotic cells, so we used polystyrene micro-beads $\left(\rho_{\text {bead }}=1,050 \mathrm{~kg} / \mathrm{m}^{3}, \quad r_{\text {bead }}=5 \mu \mathrm{m}\right)$ and adjusted the salt concentration to $0.8 \mathrm{M}\left(\rho_{\mathrm{KCl}}=1,047 \mathrm{~kg} /\right.$ $\mathrm{m}^{3}$ ) so as to be in the working conditions discussed in Sect. 2.5.

Even in these conditions a single vortex is observed in all the measurements, while its orientation is different in different experiments. The typical dynamics of the vortex frontline in $\mathrm{KCl} 0.8 \mathrm{M}$ are shown in Fig. 10. The frontline descends at an approximately constant speed, much as in Fig. 7, which supports the interpretation that the vortex reaches deeper regions.

The velocity of vortex generation $\left(v_{\mathrm{g}}\right)$ is calculated by linear regression as in Sect. 3.2:

$v_{\mathrm{gKCl}} \sim 7.1 \mu \mathrm{m} / \mathrm{s}$

The distribution of the velocities $v_{\text {up }}$ and $v_{\text {down }}$ of the two opposite flow regions once the vortex is fully developed is calculated as in Sect. 3.3 and is shown in 


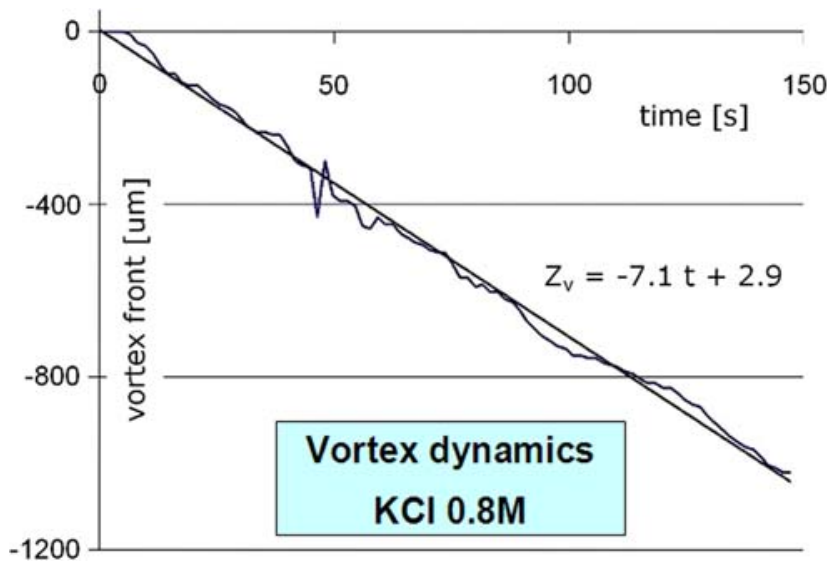

Fig. 10 Dynamics of vortex generation in $\mathrm{KCl} 0.8 \mathrm{M}$

Fig. 11. The mean value of the measured ascending stream velocities in the central portion of the flow region $\left(v_{\mathrm{up}}\right)$ is $50.0 \mu \mathrm{m} / \mathrm{s}$, while the mean value of the descending ones $\left(v_{\text {down }}\right)$ is $40.5 \mu \mathrm{m} / \mathrm{s}$.

As described in Sect. 2.5, what we measure is a combination of the flows in the solution with the gravitational fall of the particles. The gravitational fall of polystyrene micro-beads in $0.8 \mathrm{M} \mathrm{KCl}(\sim 1 \mu \mathrm{m} / \mathrm{s})$ is negligible with respect to the measured flow velocities, which are in the order of several tens of micrometers per second. In a stable condition, i.e., when the vortex is formed, the difference between $v_{\text {up }}$ and $v_{\text {down }}$ of the particles can be explained by a net ascending flow due to evaporation.

These experiments, performed at a higher salt concentration, prove that an increase in salinity has a significant impact on the generation of convection flows. The vortex frontline velocity increases from 1 (PBS) to $7 \mu \mathrm{m} / \mathrm{s}(0.8 \mathrm{M}$ $\mathrm{KCl}$ ), and the characteristic vortex speeds increase as well by a factor approximately equal to five.

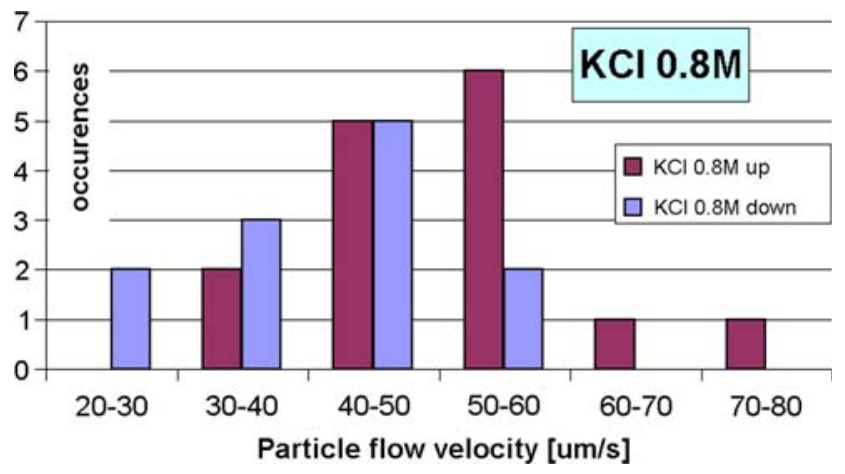

Fig. 11 Histogram of particle velocities in $\mathrm{KCl} 0.8 \mathrm{M}$ measured in the central portion of the ascending and descending flow regions. The red tiles represent particles moving upward, and the blue ones represent particles moving downward

\section{Discussion}

\subsection{Interpretation of the experimental results}

The object of the experiments is to observe the influence that evaporation at the outlet exerts on a particle inside a vertical micro-channel. In all the experiments the generation of a single vortex is observed. Although a comprehensive explanation of the cause of the vortex is outside the scope of this paper, in this section, we describe some rationales that may contribute to the explanation of this phenomenon.

As mentioned in Sect. 1, convection flows have been described for different pure fluids, which result in characteristic toroidal fields (Buffone et al. 2004. An exception to this is the study of horizontal channels, where gravity can break the symmetry and cause asymmetric flows (Buffone et al. 2005). Very low and unwanted temperature asymmetry around the outlet may also induce a significant asymmetry in the Marangoni flow field (Wang et al. 2008). In such cases, the toroidal field is substituted by a single vortex (Wang et al. 2008; Braunsfurth and Homsy 1997).

Our experiments are performed in vertical channels, without any evident asymmetry, and unexpectedly show a behavior similar to horizontal capillaries filled with a pure fluid: a single vortex develops from the meniscus region. Moreover, the vortex is inhibited with inhibited evaporation, and its dynamics closely depend on the salinity of the solution. Possible interpretations of this phenomenon are correlated with density currents and surface tension gradients at the meniscus.

Density currents can be induced by temperature/concentration gradients due to evaporation or by a small external temperature gradient around the channel. In the first case, evaporation increases the local salt concentration and decreases the local temperature close to the meniscus with respect to the bulk. The mass density of the solution in the upper region is therefore higher than that of the lower region. Density currents can be induced to level this unbalance. In the latter case, a small undesired horizontal temperature gradient in the PDMS device can be present. In principle, this would cause a mass density gradient and the resulting density currents. However, we believe that this phenomenon is not a candidate as the driving force for the vortex development: the vortex is absent when evaporation is inhibited, and its dynamics strongly depend on salt concentration. An external temperature gradient could still be the cause of asymmetry, and could decide the direction the vortex forms in.

Another possible interpretation is that a Marangoni convection is present and is intensified by the presence of inorganic solutes. Solutes do indeed have a higher local concentration where evaporation is higher, and contribute to the surface tension gradient, as shown below. This 
effect, approximately proportional to salt concentration, could make the symmetric toroidal field unstable, and is a good candidate as the driving force for vortex dynamics.

It is well known that convective flows are generated by the Marangoni effect, where a gradient in surface tension at the meniscus is caused by a gradient in temperature (Eq. 6), produced by non-homogeneous evaporation:

$\tau_{r}=-\frac{d \sigma}{d r}=-\frac{d \sigma}{d T} \frac{\partial T}{\partial r}$

where $r$ is the interfacial tangential coordinate, $\tau_{r}$ is the tangential force, and $\sigma$ is the surface tension, which in pure solutions depends only on $T$, the local temperature at the meniscus. In the case of saline solutions, surface tension also depends on the local salt concentration and can be approximated to a linear function of temperature and solute concentration (Eq. 7) (Zhang et al. 2007; Tuckermann 2007):

$\sigma(T, c)=\sigma_{0}-\sigma_{T}\left(T-T_{0}\right)+\sigma_{c}\left(c-c_{0}\right)$

where $c_{0}$ and $T_{0}$ are the reference concentration and the temperature at which $\sigma_{0}$ is measured, and the values for $\sigma_{T}$ and $\sigma_{c}$ are in the order of 0.1 dyne $\mathrm{cm}^{-1} \mathrm{~K}^{-1}$ and 1 dyne $\mathrm{L} \mathrm{cm}^{-1} \mathrm{~mol}^{-1}$, respectively, for water-based inorganic solutions. Close to the meniscus edges the temperature reaches its minimum, and the local solute concentration reaches its maximum. Hence, the minus and plus signs in front of the temperature and concentration terms in Eq. 7 indicate that the two effects add up.

The contribution of the solute effect to surface-tension fluctuations, compared to that of the thermal effect (ratio of the contributions of the thermal and concentration terms to the surface-tension gradient), is given by the product of the Soret number $X$ (Eq. 8) and the inverse Lewis number $L^{-1}$ (Eq. 9), defined as:

$\mathrm{X}=\frac{s c_{0} \sigma_{c}}{\sigma_{T}}$

$L^{-1}=\frac{\kappa}{D}$

where $s \quad\left(\sim 10^{-3} \mathrm{~K}^{-1}\right)$ is the Soret coefficient, $\kappa$ $\left(\sim 10^{-3} \mathrm{~cm}^{2} \mathrm{~s}^{-1}\right)$ is the thermal diffusivity of the fluid and $D$ $\left(\sim 10^{-5} \mathrm{~cm}^{2} \mathrm{~s}^{-1}\right)$ is the mass diffusivity of the solute. The Soret number is $\sim 10^{-3}$ for PBS and $\sim 10^{-2}$ for $\mathrm{KCl} 0.8 \mathrm{M}$ and represents the ratio of the concentration-induced surface-tension gradient to the temperature-induced surfacetension gradient. The inverse Lewis number is $\sim 10^{2}$ for both solutions and represents the heat versus mass transfer.

In the case of PBS the product $X L^{-1}$ is $\sim 10^{-1}$, while it increases to $\sim 1$ in the case of $\mathrm{KCl} 0.8 \mathrm{M}$. This supports the interpretation that the increase in the measured vortex velocities with saline concentration depends on the growing contribution of the concentration term which adds to the thermal term in inducing convection flows.
4.2 Guidelines for control of cell position inside micro-wells

The experimental results described in the previous sections are relevant to the design of biochips that require the cell position to be maintained stable. In open-outlet microfluidic systems a net upward flow due to evaporation is observed, but this cannot be used to counteract the gravitational fall of cells, since a convection pattern formed by an ascending and a descending flow is generated in the micro-channel. Hence the external trapping forces must be strong enough to counteract not only the gravitational force, but also the convection drag. The alternative is to avoid evaporation, which eliminates convection patterns throughout the channel.

As an example, the external force required in our system with PBS solution to keep eukaryotic cells stable (Sects. 3.3, 3.4) increases from $k v_{\text {grav }}$, when only the cell's gravitational fall velocity is considered ( $v_{\text {grav }} \sim 1.8 \mu \mathrm{m} / \mathrm{s}$ ), to $k\left(v_{\text {grav }}+v_{\text {sol, down }}\right)=k v_{\text {down }}$, when the downward velocity $\left(v_{\text {down }} \sim 9 \mu \mathrm{m} / \mathrm{s}\right)$ due to both the vortex velocity and the gravitational fall is considered. Therefore, the presence of convection patterns increases the required force by a factor approximately equal to five. Nevertheless, successful trapping of single cells close to the meniscus of micro-wells is possible. Dielectrophoretic forces can be used for this purpose, as demonstrated for example in (Bocchi et al. 2009). Applying an AC electric field, the time-averaged dielectrophoretic force (DEP) (Morgan and Green 2003) must counteract not only gravitational force, but also convection drag:

$$
\begin{aligned}
\left\langle F_{\mathrm{DEP}}\right\rangle= & \pi \varepsilon_{\mathrm{sol}} r_{\mathrm{part}}^{3} \operatorname{Re}\left[\frac{\varepsilon_{\text {part }}^{*}-\varepsilon_{\text {sol }}^{*}}{\varepsilon_{\text {part }}^{*}+2 \varepsilon_{\mathrm{sol}}^{*}}\right] \nabla|E|^{2} \\
& \geq\left(6 \pi \eta_{\text {sol }} r_{\text {part }}\right) v_{\text {sol }}+g \frac{4 \pi r_{\text {part }}^{3} \Delta \rho}{3}
\end{aligned}
$$

where $\varepsilon_{\mathrm{sol}}$ is the solution permittivity, $\varepsilon_{\mathrm{sol}}^{*}$ and $\varepsilon_{\text {part }}^{*}$ the complex permittivity of solution and particle, respectively, and $E$ the electric field.

The convective term is proportional to $r_{\text {part }}$, while $F_{\text {DEP }}$ and gravity are proportional to $r_{\text {part }}^{3}$, so that the relative contribution of convection is more significant for smaller particles and the minimum required intensity of the electric field increases as the cell radius decreases.

\section{Conclusions}

This article presents an investigation of the convection flows induced by evaporation inside open outlet vertical microfluidic channels filled by saline solutions. With a custom-built device based on three mirrors and a single 
microscope, it is possible to reconstruct the $3 \mathrm{D}$ position in time of particles floating in a micro-channel.

Once the channel is filled, a single vortex is generated and invades the whole channel several millimeters below the meniscus. Both the vortex generation and its characteristic speeds are measured. This phenomenon disappears when evaporation is inhibited, which demonstrates that evaporation is a direct cause. An increase in dissolved inorganic salts increases both the speed at which the vortex invades the channel and the pattern speed, suggesting that solute concentration plays a role, which can be qualitatively explained by the Marangoni theory and by density currents.

This paper contributes to understanding the effects that evaporation exerts on the particle position inside a vertical micro-channel filled with a saline solution and it provides useful information for the design of biochips that require the position of biological material to be controlled.

Acknowledgments This work was developed in the framework of the CoChiSe European Project (project reference: 034534) within the FP6 program.

Open Access This article is distributed under the terms of the Creative Commons Attribution Noncommercial License which permits any noncommercial use, distribution, and reproduction in any medium, provided the original author(s) and source are credited.

\section{References}

Abonnenc M, Altomare L, Baruffa M, Ferrarini V, Guerrieri R, Iafelice B, Leonardi A, Manaresi N, Medoro G, Romani A, Tartagni M, Vulto P (2005) Teaching cells to dance: the impact of transistor miniaturization on the manipulation of populations of living cells. Solid State Electron 49(5):674-683

Bocchi M, Lombardini M, Faenza A, Rambelli L, Giulianelli L, Pecorari N, Guerrieri R (2009) Dielectrophoretic trapping in microwells for manipulation of single cells and small aggregates of particles. Biosens Bioelectron 24:1177-1183. doi:10.1016/j. bios.2008.07.014

Braunsfurth MG, Homsy GM (1997) Combined thermocapillarybuoyancy convection in a cavity. Part II. An experimental study. Phys Fluids 9(5):1277-1286

Buffone C, Sefiane K, Christy JRE (2004) Experimental investigation of the hydrodynamics and stability of an evaporating wetting film placed in a temperature gradient. Appl Therm Eng 24:1157-1170

Buffone C, Sefiane K, Christy JRE (2005) Experimental investigation of self-induced thermocapillary convection for an evaporating meniscus in capillary tubes using micro-particle image velocimetry. Phys Fluids 17(5):Article 052104

Chamarthy P, Dhavaleswarapu HK, Garimella SV, Murthy JY, Werely ST (2008) Visualization of convection patterns near an evaporating meniscus using $\mu$ PIV. Exp Fluids 44(3):431-438

Chiou PY, Ohta AT, Wu MC (2005) Massively parallel manipulation of single cells and microparticles using optical images. Nature 436:370-372

Desai JP, Pillarisetti A, Brooks AD (2007) Engineering approaches to biomanipulation. Annu Rev Biomed Eng 9:35-53
Gazzola D, Iafelice B, Jung E, Franchi Scarselli E, Guerrieri R (2008) An integrated electronic meniscus sensor for measurement of evaporative flow. Sensors Actuators A Phys 145:194-200

Jeong OC, Konishi S (2009) Experimental study on a single particle trap with a pneumatic vibrator matrix. Microfluid Nanofluid 6:139-144. doi:10.1007/s10404-008-0318-0

Juncker D, Schmid H, Drechsler U, Wolf H, Wolf M, Michel B, de Rooij N, Delamarche E (2002) Autonomous microfluidic capillary system. Anal Chem 74:6139-6144

Kamotani Y, Ostrach S, Pline A (1994) Analysis of velocity data taken in surface-tension driven convection experiment in microgravity. Phys Fluids 6(11):3601-3609

Leitz G, Fallman E, Tuck S, Axner O (2002) Stress response in Caenorhabditis elegans caused by optical tweezers: wavelength, power, and time dependence. Biophys J 82:2224-2231

Lettieri G, Dodge A, Boer G, de Rooij NF, Verpoorte E (2003) A novel microfluidic concept for bioanalysis using freely moving beads trapped in recirculating flows. Lab Chip 3(1):34-39

Leu TS, Chen HY, Hsiao FB (2005) Studies of particle holding, separating, and focusing using convergent electrodes in microsorters. Microfluid Nanofluid 1:328-335

Morgan H, Green NG (2003) AC electrokinetics: colloids and nanoparticles. Research Studies Press Ltd, UK

Nahmias Y, Schwartz RE, Verfaillie CM, Odde DJ (2005) Laserguided direct writing for three-dimensional tissue engineering. Biotechnol Bioeng 92(2):129-136

Namasivayam V, Larson RG, Burke DT, Burns MA (2003) Transpiration-based micropump for delivering continuous ultra-low flow rates. J Micromech Microeng 13:261-271

Neuman KC, Chadd EH, Liou GF, Bergman K, Block SM (1999) Characterization of photodamage to Escherichia coli in optical traps. Biophys J 77:2856-2863

Park MC, Hur JY, Kwon KW, Park S, Suh KY (2006) Pumpless, selective docking of yeast cells inside a microfluidic channel induced by receding meniscus. Lab Chip 6:988-994

Rasmussen MB, Oddershede LB, Siegumfeldt H (2008) Optical tweezers cause physiological damage to Escherichia coli and Listeria bacteria. Appl Environ Microbiol 74(8):2441-2446

Seger-Sauli U, Panayiotou M, Schnydrig S, Jordan M, Renaud P (2005) Temperature measurements in microfluidic systems: heat dissipation of negative dielectrophoresis barriers. Electrophoresis 26(11):2239-2246

Shackman JG, Munson MS, Ross D (2007) Temperature gradient focusing for micro channel separations. Anal Bioanal Chem 387(1): 155

Thielecke H, Impidjati HT, Zimmermann H, Fuhr GR (2005) Gentle cell handling with an ultra-slow instrument: creep-manipulation of cells. Microsyst Technol 11:1230-1241

Tuckermann R (2007) Surface tension of aqueous solutions of watersoluble organic and inorganic compounds. Atmos Environ 41:6265-6275

Wang H, Murthy JY, Garimella SV (2008) Transport from a volatile meniscus inside an open microtube. Int J Heat Mass Transfer 51(11):3007-3017

Ward CA, Duan F (2004) Turbulent transition of thermocapillary flow induced by water evaporation. Phys Rev E 69(5):056308

Yang L, Banada PP, Bhunia AK, Bashir R (2008) Effects of dielectrophoresis on growth, viability and immuno-reactivity of Listeria monocytogenes. J Biol Eng 2:6

Zhang J, Behringer RP, Oron A (2007) Marangoni convection in binary mixtures. Phys Rev E 76(1):016306

Zimmermann M, Schmid H, Hunziker P, Delamarche E (2007) Capillary pumps for autonomous capillary systems. Lab Chip $7: 119-125$ 